\title{
Measurement of cross sections and properties of the Higgs boson in decays to four leptons using the ATLAS detector
}

\author{
Oliver Kortner on behalf of the ATLAS Collaboration* \\ Max-Planck-Institut für Physik, Föhringer Ring 6, 80805 Munich, Germany \\ E-mail: Oliver.Kortner@CERN.CH
}

\begin{abstract}
The analysis of the production of the Higgs boson at CERN's Large Hadron Collider provides sensitivity to the couplings of the Higgs boson to Standard Model particles and to contributions from physics beyond the Standard Model of electroweak interactions. In this note the Higgs boson production is investigated in the $H \rightarrow Z Z \rightarrow 4 \ell$ channel. A dataset of $79.8 \mathrm{fb}^{-1}$ of $13 \mathrm{TeV}$ $p p$ collision data was used to study the Higgs boson production cross section differential in the transverse momentum of the Higgs boson and the number of jets in the final state and to measure the Higgs boson production modes in the simplified templated cross section framework. All results are in agreement with the predictions of the Standard Model prediction. The measurement of the off-shell production of Higgs bosons is also found to be compatible with Standard Model prediction. An upper limit on the off-shell signal strength of 3.8 and the natural width of the Higgs boson of $14.4 \mathrm{MeV}$ could be set at $95 \%$ confidence level with $36.1 \mathrm{fb}^{-1}$ of $p p$ collision data.
\end{abstract}

ICHEP 2018, 39th International Conference on High-Energy Physics

July 4-11, 2018

Seoul, South Corea

${ }^{*}$ Speaker. 


\section{Introduction}

The $p p$ collision data collected by the ATLAS experiment [1] at the Large Hadron Collider (LHC) at a centre-of-mass energy of $13 \mathrm{TeV}$ allow for several studies of the properties of the Higgs boson. The detection of the Higgs boson through its decay into 4 charged leptons (electrons or muons) via two intermediate $Z$ bosons allows for the precise measurement of the mass and the fourmomentum of the Higgs boson event by event. This makes it possible not only to measure the mass of the Higgs boson accurately, but also to investigate the production and the decay of the Higgs boson in great detail.

At the LHC the Higgs boson is mainly produced in gluon fusion with a cross section of $49 \mathrm{pb}$. The second most frequent production process with a cross section of $3.8 \mathrm{pb}$ is the production of the Higgs boson in vector boson fusion where the two colliding quarks radiate off intermediate $W$ or $Z$ bosons which collide and create a Higgs boson. Higgs bosons are produced in association with a real $W$ or $Z$ boson by Higgs radiation with a slightly smaller cross section of $2.2 \mathrm{pb}$. Very rarely Higgs bosons are produced in association with a top-antitop quark pair ( $0.5 \mathrm{pb}$ cross section). The $79.8 \mathrm{fb}^{-1}$ of $p p$ collision data recorded by the ATLAS experiment in the years 2015 to 2017 provide sensivity to all four production processes and allow for the measurement of differential Higgs boson production cross sections in the $H \rightarrow 4 \ell$ channel.

In this note the measurement of the Higgs boson production cross section differential in the transverse momentum of the Higgs boson and differential in the number of jets in the final state is studied. The distribution of the Higgs boson's $p_{\mathrm{T}}$ depends on the number of initial-state-radiation jets in the gluon-gluon fusion process and therefore provides a test of the prediction of perturbative QCD. The distribution is also sensitive to the Langrangian structure of the Higgs boson interactions. The measurement of the multiplicity of jets in the final state tests the theoretical modelling of gluon emission and depends on the fractions of the different production modes.

The main focus of this note is on the measurements of the different production modes in the simplified template cross section framework which tries to map the event topologies at reconstruction level to corresponding topologies at particle level.

A smaller fraction of the collected data, $36.1 \mathrm{fb}^{-1}$, was used to measure the off-shell production of the Higgs boson which gives access to the small natural width of the Higgs boson which can be understood from the following simple consideration. Let $\sigma_{o f f-\text { shell }}^{g g \rightarrow H^{*} \rightarrow Z Z}$ denote the measured off-shell cross section and $\sigma_{o f f-s h e l l, S M}^{g g \rightarrow H^{*} \rightarrow Z Z}$ the Standard Model prediction for this cross section. The ratio of $\sigma_{o f f-\text { shell }}^{g g \rightarrow H^{*} \rightarrow Z Z}$ and $\sigma_{o f f-\text { shell,SM}}^{g g \rightarrow H^{*} \rightarrow Z Z}$, the so-called "off-shell signal strength" $\mu_{o f f-\text { shell }}$, can be expressed as the product of correction factors $\kappa_{g, o f f-s h e l l}^{2}$ and $\kappa_{Z, o f f-s h e l}^{2}$ to the production and the decay respectively:

$$
\mu_{o f f-s h e l}=\frac{\sigma_{o f f-\text { shell }}^{g g \rightarrow H^{*} \rightarrow Z Z}}{\sigma_{o f f-\text { shell }, S M}^{g g \rightarrow H^{*} \rightarrow Z Z}}=\kappa_{g, o f f-\text { shell }}^{2} \cdot \kappa_{Z, o f f-\text { shel }}^{2} .
$$

A similar equation holds for the on-shell production:

$$
\mu_{\text {on-shel }}=\frac{\sigma_{o f f-\text { shell }}^{g g \rightarrow H \rightarrow Z Z}}{\sigma_{o f f-\text { shell }, S M}^{g g \rightarrow H \rightarrow Z Z}}=\frac{\kappa_{g, \text { on-shell }}^{2} \cdot \kappa_{Z, \text { on-shel }}^{2}}{\Gamma_{H} / \Gamma_{H}^{S M}}
$$


where $\Gamma_{H} / \Gamma_{H}^{S M}$ is the ratio of the actual and the predicted natural width of the Higgs boson. If the corrections to the couplings $\kappa_{o f f-s h e l l}$ and $\kappa_{o n-s h e l l}$ are the same, the width of the Higgs boson can be measured via the ratio of the on-shell and the off-shell signal strengths:

$$
\frac{\mu_{\text {on-shell }}}{\mu_{\text {off-shell }}}=\frac{\Gamma_{H}}{\Gamma_{H}^{S M}}
$$

\section{Event selection}

For the study of the on-shell Higgs boson production [3] events with two pairs of oppositely charged isolated electrons or muons from a common vertex were selected. The mass $m_{12}$ of one lepton pair was required to be close to the mass of the $Z$ boson: $50 \mathrm{GeV}<m_{12}<106 \mathrm{GeV}$. The mass of the 4 lepton system was required to be between $118 \mathrm{GeV}$ and $129 \mathrm{GeV}$. The electron candidates are reconstructed from ID tracks that are matched to energy clusters in the electromagnetic calorimeter. In $|\eta|<2.5$ the muon candidates were reconstructed from tracks in the inner detector which could be match to a muon spectrometer track. For $2.5<|\eta|<2.7$ muon spectrometer tracks were used for the muon identification.

Apart from the requirement on the four-lepton invariant mass $m_{4 \ell}$, which was altered to $220 \mathrm{GeV}<$ $m_{4 \ell}<2000 \mathrm{GeV}$, and the requirement that both lepton pairs must have a mass close to the $Z$ boson mass, the same selection was used for the measurement of the off-shell production cross section [4]. In order to enlarge the data sample also decays of one $Z$ boson into a pair of neutrinos were allowed. The presence of neutrinos leads to a significant missing transverse energy which was required to be greater than $175 \mathrm{GeV}$.

\section{Study of the Higgs boson production}

Table 1 summarizes the measured fiducial Higgs-boson production cross sections for the $H \rightarrow 4 \ell$ channel. There is a $14 \%$ excess in the number of observed events due to an upward fluctuation of $H \rightarrow 2 e 2 \mu$ events which leads to a larger measured cross section than the predicted. Yet, the measured cross sections agree with the values predicted by the Standard Model within the experimental and theoretical uncertainties. The dominant experimental systematic uncertainties comes from a $3 \%$ uncertainty on the luminosity measurement and a 4 to $8 \%$ uncertainty from pile-up which mainly affects the lepton isolation.

The dependence of the measured differential fiducial cross section on he transverse momentum of the Higgs boson on the left and the fiducial cross section as a function of the number of jets in the final state was also measured. No statistically significant deviations of the differential distributions from the Standard Model predictions have been found.

Figure 1 summarizes the comparisons of the measured cross sections for the different production modes with the Standard Model predictions. There is excellent agreement of the measured cross sections for gluon fusion and $V H$ production. The measured cross section for the vector boson fusion production exceeds the prediction by almost a factor 3, yet this deviation has only a significance of 1.8 standard deviations. No events in the $t t H$ category were observed so that only an upper $95 \%$ confidence limit on the corresponding cross section could be set. This limit is in agreement with the Standard Model prediction. The comparison of the measured cross sections 
Table 1: Comparison of the measured with the predicted fiducial cross sections [3]. The leading lepton pair, i.e. the lepton pair with the largest invariant mass is denoted first in the subscript of the cross section symbol.

\begin{tabular}{lcccc}
\hline \hline Cross section $[\mathrm{fb}]$ & Data ( & \pm (stat.) & \pm (syst.) $)$ & Standard Model prediction \\
\hline$\sigma_{4 \mu}$ & 0.97 & \pm 0.17 & \pm 0.05 & $0.886 \pm 0.039$ \\
$\sigma_{4 e}$ & 0.61 & \pm 0.21 & \pm 0.07 & $0.886 \pm 0.039$ \\
$\sigma_{2 \mu 2 e}$ & 0.88 & \pm 0.21 & \pm 0.08 & $0.786 \pm 0.035$ \\
$\sigma_{2 e 2 \mu}$ & 1.37 & \pm 0.22 & \pm 0.07 & $0.786 \pm 0.035$ \\
\hline \hline$\sigma_{\mathrm{tot}}[\mathrm{pb}]$ & 67.2 & \pm 6.8 & \pm 4.1 & $55.7 \pm 2.5$ \\
\hline
\end{tabular}
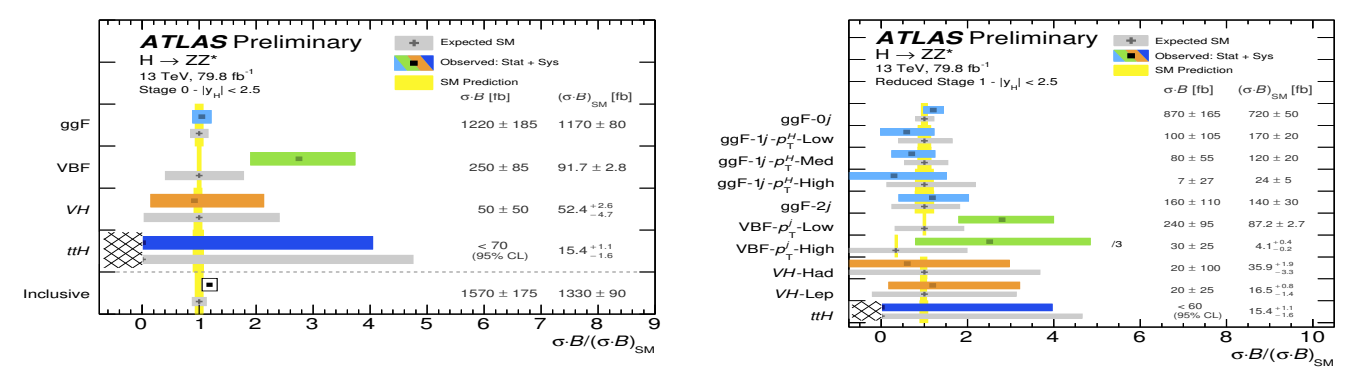

Figure 1: Comparison of the measured cross sections for the different production modes with the Standard Model predictions [3] for the four main production modes on the left and finer categories on the right.

in more resolved final state categories with the theoretical predictions also shows no statistically significant deviation from the Standard Model.

\section{Measurement of the off-shell Higgs boson production}

The measurement of the off-shell Higgs boson production cross section revealed no excess over the dominant $Z Z$ and $W Z$ background such that only an upper $95 \%$ confidence limit on the off-shell signal strength of 3.8 and on the natural width of the Higgs boson of $14.4 \mathrm{MeV}$ compatible with the Standard Model prediction of $4 \mathrm{MeV}$ could be set.

\section{References}

[1] ATLAS Collaboration, "The ATLAS Experiment at the CERN Large Hadron Collider," JINST, vol. 3, p. S08003, 2008.

[2] ATLAS Collaboration, "Measurements of the Higgs boson production, fiducial and differential cross sections in the $4 \ell$ decay channel at $\sqrt{s}=13 \mathrm{TeV}$ with the ATLAS detector," Tech. Rep. ATLAS-CONF-2018-018 (https://cds.cern.ch/record/2621479), CERN, Geneva, Jun 2018.

[3] ATLAS Collaboration, "Constraints on off-shell Higgs boson production and the Higgs boson total width in $Z Z \rightarrow 4 \ell$ and $Z Z \rightarrow 2 \ell 2 v$ final states with the ATLAS detector," 2018. 